\title{
La influencia de las 4Ps en el Marketing Digital para la Cooperativa de Ahorro y Crédito JEP
}

\section{The influence of 4Ps on Digital Marketing for the Cooperativa de Ahorro y Crédito JEP}

\author{
Elizabeth Magdalena Trelles Méndez \\ emtrellesm@psg.ucacue.edu.ec \\ Universidad Católica de Cuenca, Cuenca \\ Ecuador \\ https://orcid.org/0000-0001-8178-3278 \\ Juan Carlos Erazo Álvarez \\ jcerazo@ucacue.edu.ec \\ Universidad Católica de Cuenca, Cuenca \\ Ecuador: \\ https://orcid.org/0000-0001-6480-2270 \\ Cecilia Ivonne Narváez Zurita \\ inarvaez@ucacue.edu.ec \\ Universidad Católica de Cuenca, Cuenca \\ Ecuador: \\ https://orcid.org/0000-0002-7437-9880
}

Recibido: 10 de agosto de 2019

Aprobado: 30 de agosto de 2019

\section{RESUMEN}

El Marketing es una de las disciplinas con mayor campo de acción en el mundo profesional, se enfoca en entender las necesidades de un público con la finalidad de impulsar las ventas. Llega hasta la forma adecuada de comunicación para crear un vínculo entre las personas y la marca. La Unidad de análisis de esta investigación es la Cooperativa de Ahorro y Crédito "Juventud Ecuatoriana Progresista" Ltda., (JEP) cuyo problema es la deficiente colocación de tarjetas de crédito que se originan principalmente por ser un producto que está en el ciclo de desarrollo, para mejorar esta situación se plantean estrategias de marketing digital enfocadas en las 4Ps. La Investigación tiene un enfoque mixto, es de tipo exploratoria y descriptiva, no experimental y probabilística y según su 
alcance es descriptiva. Como resultados de la investigación se evidenció falencias especialmente en la gestión digital lo que no permite dar una respuesta eficaz y a lo que se plantean estrategias en función al tipo de organización.

Descriptores: Marketing; Digital; Estrategia; Cooperativa; Tarjetas de crédito.

\begin{abstract}
Marketing is one of the disciplines with the greatest field of action in the professional world, it focuses on understanding the needs of an audience in order to boost sales. It reaches the proper form of communication to create a link between people and the brand. The unit of analysis of this research is the Cooperativa de Ahorro y Crédito "Juventud Ecuatoriana Progresista" Ltda., (JEP) whose problem is the poor placement of credit cards that originate mainly as a product that is in the development cycle to improve this situation, digital marketing strategies focused on 4Ps are proposed. The Research has a mixed approach, it is exploratory and descriptive, non-experimental and probabilistic and according to its scope is descriptive. As results of the investigation, there were flaws, especially in digital management, which does not allow for an effective response and to which strategies are proposed depending on the type of organization.
\end{abstract}

Descriptors: Marketing; Digital; Strategy; Cooperative; Credit cards.

\title{
INTRODUCCIÓN
}

La unidad de análisis de esta investigación es la Cooperativa de Ahorro y Crédito "Juventud Ecuatoriana Progresista" Ltda., (JEP) que es una entidad dedicada a las finanzas sociales, creada mediante acuerdo Ministerial 3310, el 31 de diciembre de 1971 y calificada por la Superintendencia de Bancos y Seguros con Resolución SBS-2003-0596, de agosto 12 del 2003.

La Cooperativa JEP actualmente tiene más de medio millón de socios activos, que equivale a un $70 \%$ del mercado, de los cuales, la mayoría son de género femenino que están ejerciendo alguna actividad de emprendimiento en los sectores urbano marginales. 
Sus inicios fueron en la parroquia Sayausí del cantón Cuenca, ubicada en la provincia del Azuay. Son 29 jóvenes creativos y emprendedores que han logrado ser el pilar fundamental de apoyo crediticio a las zonas desatendidas de la banca tradicional, cualidad que ha caracterizado para la aceptación y confianza de la gente, esto le ha permitido a la Cooperativa alcanzar los primeros lugares dentro del ranking de las mejores cooperativas a nivel nacional y actualmente cuenta con más de 46 agencias en todo el Ecuador. El problema que pretende resolver esta investigación es la disminución en la colocación de tarjetas de crédito que se originan principalmente porque la Cooperativa en este servicio es poco competitiva dentro del nicho de mercado en la provincia del Azuay por ser un producto que está en el ciclo de desarrollo y por ser la primera Cooperativa que emite tarjetas de crédito dentro de la economía popular y solidaria. También porque los servicios de las tarjetas de crédito tienen limitaciones debido a que no están aceptados en todos los sectores empresariales y comerciales por la falta de negociación y alianzas estratégicas lo que produce que los socios prefieran ir a la competencia.

No existen encuestas sobre el servicio de las tarjetas de crédito, por lo cual no se puede hacer un seguimiento a los socios para que puedan calificar el servicio y poder mejorar con las sugerencias entregadas por el socio.

La marca y nombre de las tarjetas de crédito aún no está posicionada en la ciudad de Cuenca, al ser un servicio financiero relativamente nuevo, no se tiene aún el prestigio con el que si cuenta la competencia lo que causa que no se tenga aun el flujo de socios necesario para tener mayores ingresos. En función a esta problemática se plantea como objetivo el diseño de estrategias de marketing que permitan posicionar a las tarjetas de crédito de la Cooperativa JEP y como consecuencia mejorar sus ingresos.

\section{El Marketing, el mercado y los consumidores en la actividad comercial}

El Marketing ha sido un tema muy controversial por la amplitud que conduce a la palabra marketing para describirlo en Iberoamérica en sus distintas traducciones 
como mercadotecnia, mercadeo, mercadología, pero todas estas palabras dirigen al mismo punto que es la distribución y transmisión de propiedad de los productores a los consumidores, desde una perspectiva teórica el marketing es una ciencia social que estudia los intercambios comerciales de dos partes intervinientes, los autores concuerdan con esta definición debido a que esta definición da un panorama más claro de que es el marketing con mayor profundidad (Rivera, 2012)

El marketing tiene múltiples definiciones con distintas variables y no tiene un concepto único, pero se puede conceptualizar como una actividad que está sujeta a muchos cambios acelerados que se dan entre los clientes y las empresas en una relación comercial, lo único que cambia son los mercados de manera constante, del mismo modo, es una herramienta fundamental como plataforma para dar a conocer sus productos que puede satisfacer una o varias necesidades (Sellers, 2006) Para Córdova (2009) el marketing debe entenderse como una guía para la organización de una empresa y no como una técnica para incrementar ventas, es un sistema de pensamiento y un sistema de acción. Acota de la misma forma que es un proceso social de gestión por el cual los individuos y grupos obtienen lo que necesitan y desean, mediante creación de intercambio y valor de servicios a los demás, por lo tanto, esta técnica resulta efectiva a medida que la empresa adopta este modelo internamente a los empleados de la organización.

La evolución del concepto de marketing comienza con el período de descubrimiento en el año 1990, cuando se practicaba, en el mercado, el comercio e intercambios de valores, fenómeno conocido como macroeconómico, entonces apareció el término marketing que reemplazo a las palabras comercio, distribución e intercambio y se desarrolló la publicidad, promoción para impulsar la compra y aplicación de hallazgos psicológicos con procesos cognitivos del consumidor, la autora toma como pilar fundamental teórico la evolución del concepto de marketing de los autores descritos a continuación (Garcillán y Rivera, 2012). 
La evolución del marketing tiene su segunda etapa con el período de conceptualización en el año 1910-1920. En esta época creció la economía del mundo y la de los EEUU en su mayoría las cuales influyeron mucho para que apareciera tres enfoques de análisis; el enfoque de comodities, el funcional y el institucional, entonces apareció el concepto de creación de utilidad económica y se desarrollan los conceptos de utilidad de forma, utilidad de posesión y utilidad de consumo, la autora está de acuerdo con el análisis de los autores (Rivera y Garcillán, 2012).

La evolución del marketing en marco conceptual en las empresas. Las empresas manufactureras ha experimentado varias etapas de cambios, la primera es la era de la producción que cubre de los primeros años en EE.UU hasta el año 1920, los bienes eran escasos así que los compradores estaban dispuestos a aceptar casi cualquier bien producido y obtener el mayor provecho, en la segunda etapa la era de las ventas, los productores fabricaban más de lo que los demandantes necesitaban, y la oferta se intensifico (León, Erazo, Narváez y Solís, 2019)

La evolución del marketing tiene su tercera etapa con el período de integración en el año 1920-1930, en este periodo se integran todas las áreas del marketing y aparecen dos áreas con utilidad fundamental, la venta mayorista e investigación de mercados, la autora está de acuerdo el análisis propuesto, debido a que hay una integración de criterios que permite que aparezcan estas dos áreas, la venta mayorista que permite a una empresa el crecimiento exponencial de sus ventas y la investigación de mercados que es una análisis profundo de los gustos y preferencias de los consumidores. (Álvarez, Erazo, Narváez y Erazo, 2019)

La era de la internacionalización y el libre comercio, instaurado el internet y la era de la información digital, la nueva arquitectura tecnológica, los documentos virtuales, la marginación en los espacios virtuales, los nuevos modelos de negocios basados en personalización y marketing, se expresa un acuerdo con el criterio de una de la etapas de la evolución de los conceptos del marketing, ya que muchas varia- 
bles como la economía de los países y los cambios de las herramientas de la tecnología, impulsan al marketing sin barreras como una telaraña mundial de canales de información digital sin ninguna barrera para la comunicación (Rivera, 2015).

El proceso del marketing está compuesto de cinco pasos: Primero entender el mercado, las necesidades y los deseos de clientes. Seguidamente diseñar una estrategia de marketing con visión para el cliente. A continuación, elaborar un programa de marketing integrado que proporciona un valor superior. Del mismo modo se debe establecer relaciones redituables y lograr el deleite del cliente, finalmente debe captar valor de los clientes para obtener utilidades y activos de ellos. Los autores desarrollan una concordancia con los pasos, delimitados para el proceso del marketing, por lo tanto, es de vital importancia para las empresas conocer y entender el proceso del marketing de forma integral, aplicando los cinco pasos descritos por estos autores, se debe aplicar de la manera más sencilla, clara y muy concreta, se debe captar un valor para el socio cliente es aplicar de manera correcta esta estrategia de marketing, ya que se está captando un valor monetario intangible a futuro que recompensará con la fidelidad del socio-cliente al mantenerlo en la cartera de socios por un tiempo duradero, esta relación provechosa debe seguir dando ganancias, en la siguiente figura, lo planteado por (Kotler y Armstrong, 2012). 


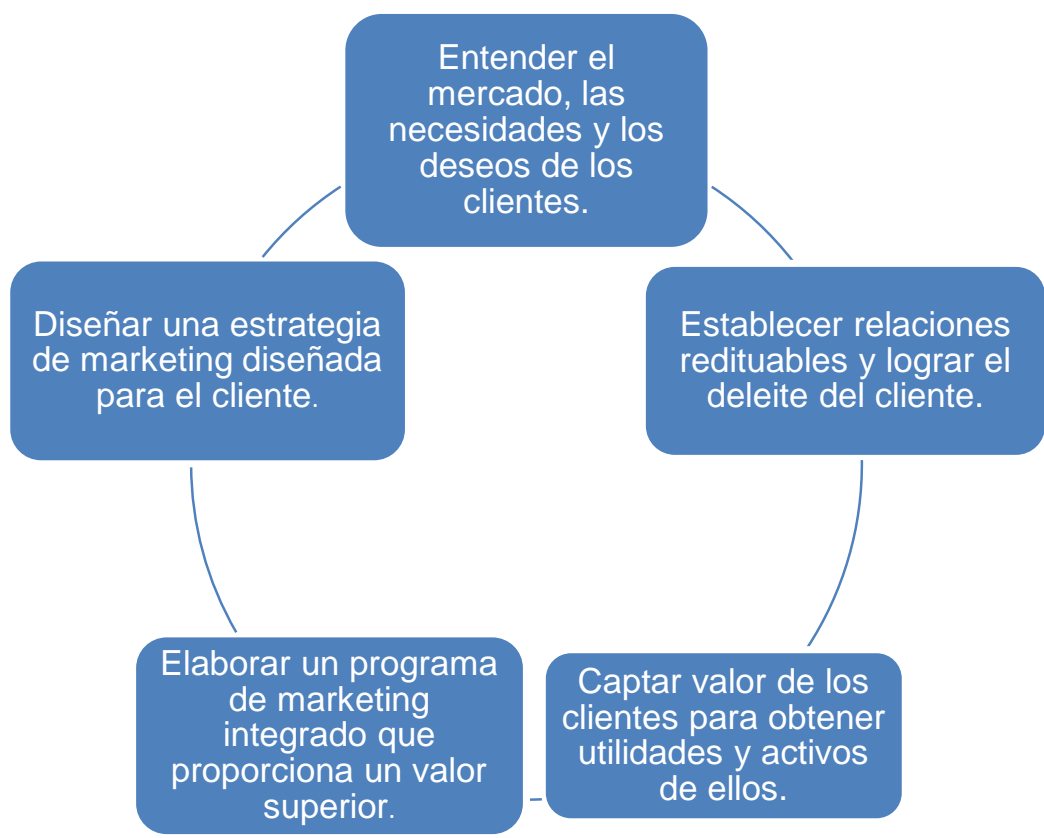

\section{La tipología del Marketing, con enfoque en el cliente}

El marketing digital es un instrumento de vital importancia para elaborar estrategias en el mercado online, debido a que no tiene barreras geográficas y el mercado objetivo es muy amplio, los costos de esta herramienta de marketing son muy bajos debido a la plataforma virtual, cada vez el marketing se va haciendo más digital, ya que las personas han dejado los vehículos tradicionales como TV y el radio, debido a que los consumidores han cambiado su manera de informarse, es una de las herramientas más completas para realizar marketing digital ya que conecta al mundo sin barreras en distintos puntos geográficos (Keller, 2009).

Marketing Offline, esta herramienta está direccionada a realizar marketing fuera del internet con métodos tradicionales como un anuncio en el periódico, distribución de merchandasing, telemarketing, cabe entender que las personas y su forma de comunicarse es a través de lo digital, la preferencia es que cada vez las personas 
toman decisiones más integradas y existe barreras entre el marketing online y offline, a la hora de comprar en las tiendas virtuales y físicas, es importante entender que las mejores experiencias para los compradores es en las tiendas físicas.

Outbound Marketing, son ejemplos citados en el marketing offline, esta herramienta se caracteriza porque la marca va detrás del consumidor como anuncio, una conversación o llamada, es la estrategia de visitar empresas para activar nuevos clientes, esta herramienta tiene sus desventajas pues siempre se interrumpe una actividad al posible cliente para ingresar con la promoción de los productos de manera directa, otra desventaja es que el cliente tiene una percepción negativa de la marca debido a la interrupción el día a día (Velásquez, 2014)

Inbound marketing, esta herramienta contraria al outbound markeitng, el inbound es un marketing que gana poder por la atracción que realiza, en lugar de invitar a los posibles consumidores, lo que realiza es la atracción de los consumidores, con un contenido bastante relevante, entonces luego convertirlos en clientes, esta herramienta de marketing no es barata por la inversión que amerita, y es conocida como marketing de atracción (García, 2013).

Marketing de contenidos, es la mezcla del marketing digital y el inbound marketing, estas son algunas formas para conseguir el éxito en el marketing en internet, una de ellas es el marketing de contenidos que lleva contenido muy relevante y valioso para el comprador, tiene el único fin de convencer a los consumidores en su día de compra para crear una apreciación auténtica de la marca, estos contenidos son brindados en diferentes herramientas como: blogs, redes sociales, emails, formatos, e-books, infográficos entre otros (Espinoza, 2013).

Marketing de redes sociales, las más usadas son twitter, facebook, instagram, Likedin, Son las cuatro redes sociales más importantes que los consumidores digitales están usando información y contenido valioso para informarse, estas redes permite interactuar con el público y observar, también las redes sociales crean un ambiente de comunicación y relación, que permite encontrar al consumidor con 
mayor precisión, pero se debe conocer y desarrollar para un marketing más eficiente (Suárez y Fernández, 2014).

Marketing de búsquedas, Search Engine marketing o SEM, son estrategias de marketing intrínsecamente de los buscadores como google o bing, estos buscadores contienen la propaganda para likes patrocinados, permite conocer con profundidad el SEO con la guía más profunda y completa, en los dos casos la marca siempre aparecerá frente al usuario, SEO (Search Engine Optimization) es la práctica de utilizar un rango de técnicas, incluidas la reescritura del código HTML, la edición de contenidos, la navegación en el site, campañas de enlaces y más acciones, con el fin de mejorar la posición de un website en los resultados.

Finalmente consideramos que dentro de la tipología del marketing es fundamental la comunicación integrada es una de las herramientas del marketing más poderosas de las empresas, esto consiste en tener claro la información sobre el consumidor final y la empresa combinando de manera armoniosa las herramientas de comunicación que faciliten el contacto con su mercado objetivo y orientan en la misma dirección para crear una relación estable y duradera para las personas que intervienen, tratando de influir sobre la percepción de la persona o personas de la propuesta de valor de la empresa.

La ciencia para dirigir los recursos de Marketing no ha avanzado considerablemente, las organizaciones quieren mejorar su efectividad y esto requiere conceptos e instrumentos para el análisis substancial que oriente a una mejora. En cualquier caso, se debe buscar una teoría de acción de Marketing, entendiendo por teoría un sistema explícito y coherente entre variables, relaciones con bases empíricas potenciales o reales, encaminado a obtener comprensión, predicción y control de un conjunto de fenómenos, es decir que se podría predecir con un histórico de efectividad y ampliar los instrumentos usados para mejorar las mismas (Kotler y Armstrong, 2012). 
La comunicación es una de las variables más importantes que tienen las empresas ya que ayuda a la comunicación asertiva entre la empresa y los consumidores, influye mucho en la percepción que se forma sobre la demanda, con esta herramienta se facilita que los consumidores se integren al mercado y adopten un comportamiento que en el caso de las empresas será el de compra, pueden ser empresas con fines de lucro o no, esta comunicación llevará a que este mercado tenga un comportamiento de compra en función a la comunicación emitida (Ardura, 2007). Esta es la filosofía que ha guiado este trabajo de investigación, observando metodológicamente una realidad, orientándose a encontrar un sistema explícito y coherente entre la misma, el esfuerzo de Marketing realizado por las organizaciones y la respuesta del mercado, para encontrar la efectividad a fin de conseguir las metas planteadas y materializar los resultados con números positivos para la entidad económica planteada (Chinguel y Caqui, 2017).

La comunicación en el proceso del marketing, es uno de los instrumentos mix que puede servir a cualquier organización, para conseguir objetivos de marketing propuestos, del mismo modo contribuye el producto como medio para satisfacer las necesidades del consumidor, el precio que aporta a la rentabilidad y los canales de distribución, también es fundamental en la comunicación conocer características y comportamientos del consumidor en el mercado, aspecto cualitativo para formar una estrategia de comunicación hacia su entorno y la competencia (Ardura, 2007). Para diseñar una adecuada línea de comunicación en cada situación que contribuya a los objetivos del marketing, se requiere especialistas en compresión global del conjunto de actividades del marketing, solo considerando los objetivos de marketing que pretende conseguir, se puede diseñar una estrategia adecuada de comunicación efectiva entre los consumidores y el resto del público interesado en el servicio o productos.

La comunicación del marketing integrado, es una herramienta del marketing que se ha utilizado durante muchos años, como modo genérico de distintos instrumentos 
de comunicación en la esfera del marketing se emplea por lo general de manera independiente, en ocasiones de forma inconexa, la dinámica actual ha impulsado muchos cambios notables que ha puesto relieve a la necesidad de una integración estratégica en los instrumentos de comunicación (Ardura, 2007).

\section{METODOLOGÍA}

La Investigación realizada tiene un enfoque mixto, es de tipo exploratoria y descriptiva, no experimental y probabilística. Parte de una exhaustiva revisión sistemática y se ancla al estudio de campo en búsqueda de la evidencia empírica. Su enfoque fue mixto donde predomina lo cualitativo, pero con elementos cuantitativos mediante el uso de la estadística descriptiva. Según su alcance fue descriptiva. La unidad de análisis es la Cooperativa de ahorro y crédito JEP de la Ciudad de Cuenca.

Se aplicó el método inductivo - deductivo puesto que consistió en la inferencia basada en la lógica y en hechos particulares, que van en un sentido deductivo de lo general a lo particular y en un sentido lógico, el deductivo de lo general a lo particular va en sentido contrario. El Analítico - Sintético porque se estudió los hechos reales partiendo de la descomposición del objeto de estudio, en cada una de las partes partiendo de un estudio en forma individual, haciendo un análisis y luego se integran dichas partes, para estudiarlas de manera integral, en síntesis. El método descriptivo sirvió para estudiar los fenómenos desconocidos, observarlos en su ambiente natural y luego dar a conocer las relaciones de subordinación y de coordinación entre los elementos que lo constituyen. El método histórico - lógico, se centró en las experiencias pasadas, indistintamente de la disciplina científica, en la actualidad este método se presenta como una búsqueda critica de la verdad que sustenta a los acontecimientos del pasado (Tamayo, 2009) 
Se realizó encuestas y entrevistas a la población objeto de estudio que está conformada por todos los socios que están utilizando nuestros servicios de la Cooperativa JEP y que constan como económicamente activos, entre las edades de 25 años hasta 68 años en la ciudad de Cuenca.

El universo total de estudio se divide en:

- Socios de las tarjetas de crédito.

- Empleados.

Teniendo un $95 \%$ de confiabilidad y un error del $5 \%$, la muestra a tratar sería la siguiente:

Población económicamente activa de Cuenca.

Tabla 1.

Total, habitantes Cuenca 712.127

Total, mujeres 375.083

Total, hombres 337.044

Pea Activa 324.665

Fuente: INEC 2017.

$$
n=\frac{Z 2 P Q N}{e 2(N-1)+Z 2 P Q}
$$

Una vez calculada la muestra se ha determinado que de un universo de 324 mil 665 de personas económicamente activas y que estén recibiendo nuestros servicios de la Cooperativa JEP, se aplicará la encuesta a 384 personas.

\section{RESULTADOS}

Entre los resultados más relevantes de la investigación de campo podemos resaltar que en el rango de 25 a 35 años se encuentra concentrado el $57 \%$ del mercado objetivo. 
Con relación al tipo de producto, que es la tarjeta de crédito el dato es revelador porque en este intervalo se ubica la mayor parte de la población económicamente activa. El segundo rango de 36 a 44 años, tiene un peso del $28 \%$ de participación. El rango de 45 años a 68 años tiene una participación del 15\%. Implica también una segmentación por edad para el desarrollo del sistema de estrategias de marketing, ya que las necesidades de financiamiento y el segmento de mercado son diferentes, la variable edad es de vital importancia para la segmentación de mercado y sus necesidades.

La actividad económica de tipo independiente se encuentra concentrada el 50\% del mercado objetivo. Con relación al tipo de producto, que es la tarjeta de crédito el dato es relevante porque en este intervalo se ubica nuestro mercado objetivo. Los empleados privados, tienen un $32 \%$ de participación y el empleado público tiene un $11 \%$, estas características serán utilizadas en el desarrollo de las estrategias de marketing.

El $77 \%$ de los socios no pueden hacer uso adecuado de las tarjetas de crédito en todos los locales comerciales en la Ciudad de Cuenca, debido a que la tarjeta no cuenta con el sistema de diferimiento, lo que provoca que el socio deba llamar al call center o acercarse a la agencia más cercana para realizar los diferidos, este aspecto definitivamente es uno de los que más impacto negativo tiene al momento de la colocación de las tarjetas en el mercado. Los socios desean diferir sus compras y en la actualidad no lo pueden hacer directamente con las casas comerciales. El $80 \%$ menciona que los canales de comercialización preferidos para recibir mensajes publicitarios son los de los medios digitales.

En función a los resultados obtenidos en la investigación se plantean las siguientes estrategias:

\section{Estrategias Cooperativa JEP.}

Figura 2. 


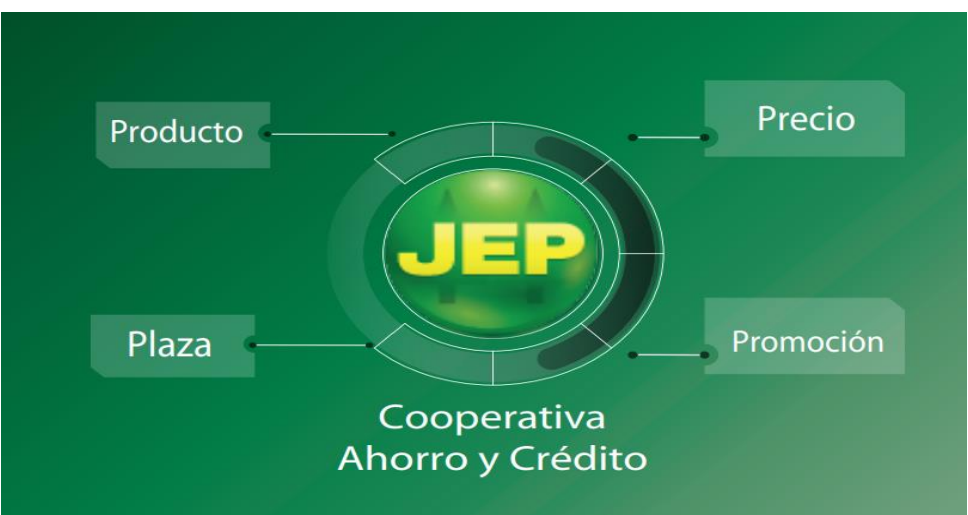

Fuente: Kotler y Armstrong (2012).

\section{Producto:}

Se plantea una innovación al servicio, de tal manera que se desarrolle en el Departamento de Investigación y desarrollo, el software para la implementación en todos los locales comerciales.

Para alcanzar el propósito de mejora de la variable diferimiento con una herramienta más versátil y convergente se recomienda implementar un módulo adicional en la JEP virtual, que se despliega en menús llamado diferimiento. 
Modelo de la herramienta de diferimiento en JEP virtual.

Figura 1.

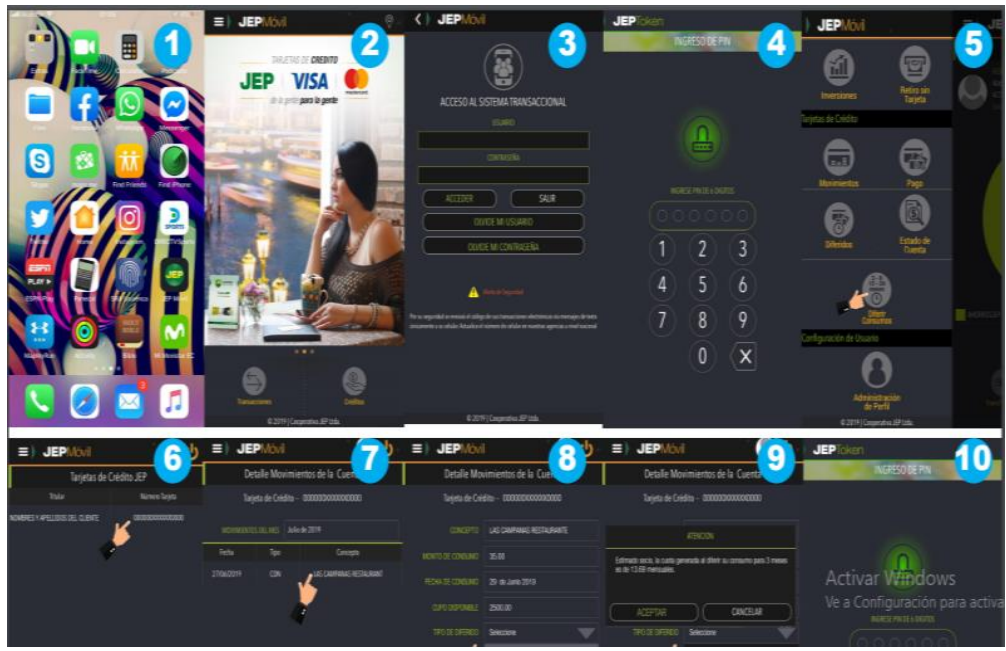

En la investigación de mercado se encontró la falencia de este servicio, no dispone la Cooperativa JEP en los teléfonos inteligentes una aplicación APP para diferir su compra, de tal forma que, para sustentar la intervención de la propuesta con relación al diferimiento, se recomienda crear una aplicación al teléfono personal de cada socio para que cuando compra acceda directamente y difiera. Aplica a todo tipo de teléfonos inteligentes.

Aplicación de App en teléfonos celulares.

Figura 4.

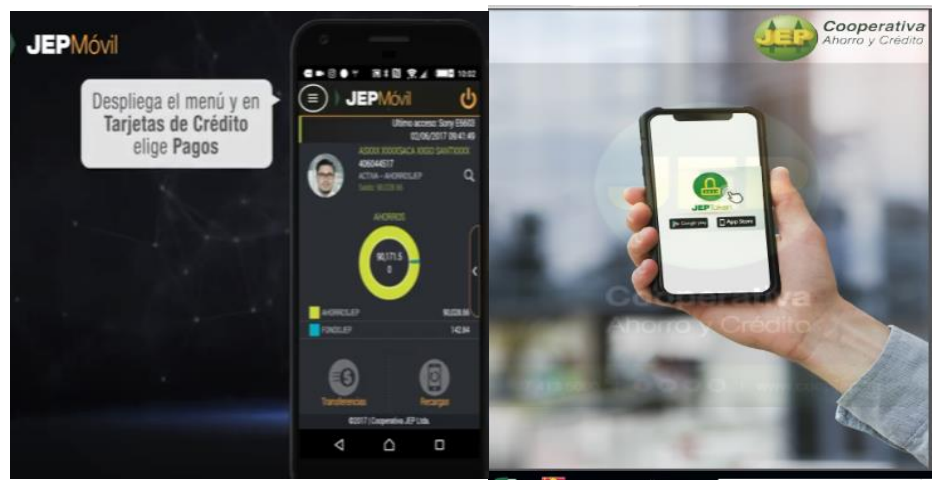

A continuación, se describe cómo funciona este sistema de diferidos mediante un flujo de procesos. 
Algoritmo del proceso del sistema de diferimiento.

Figura 52.

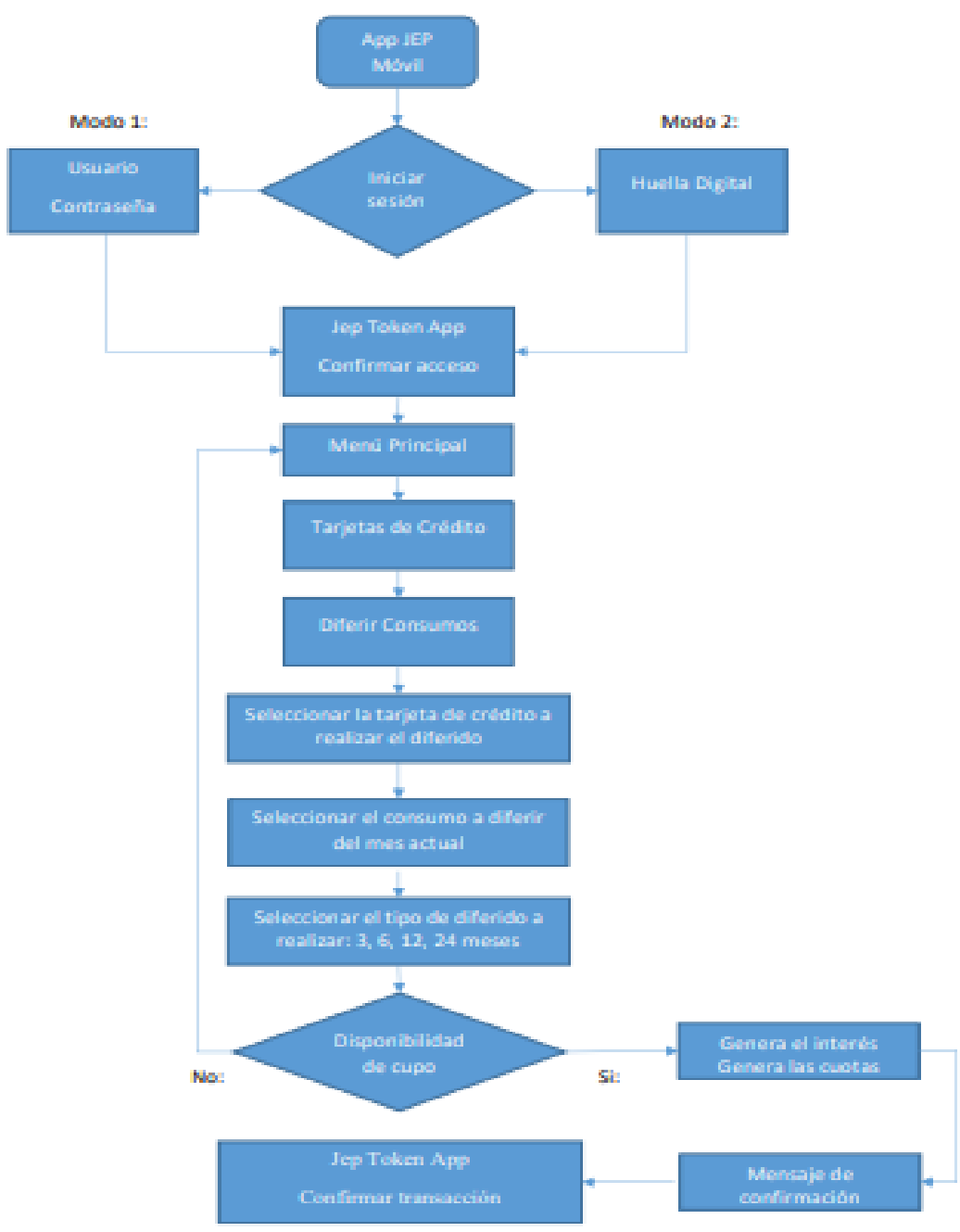

\section{Plaza:}

La plaza consiste en la macro segmentación de la innovación. En el caso de la investigación, la Cooperativa JEP tiene 46 agencias a nivel nacional, constituyendo la plaza que 
implica el canal de distribución y comercialización. Tratándose de un servicio, la estrategia definida: Transferencia del conocimiento de la innovación a nivel de los funcionarios ejecutivos y operativos de las Agencias.

Transferencia de conocimiento.

Figura 6.

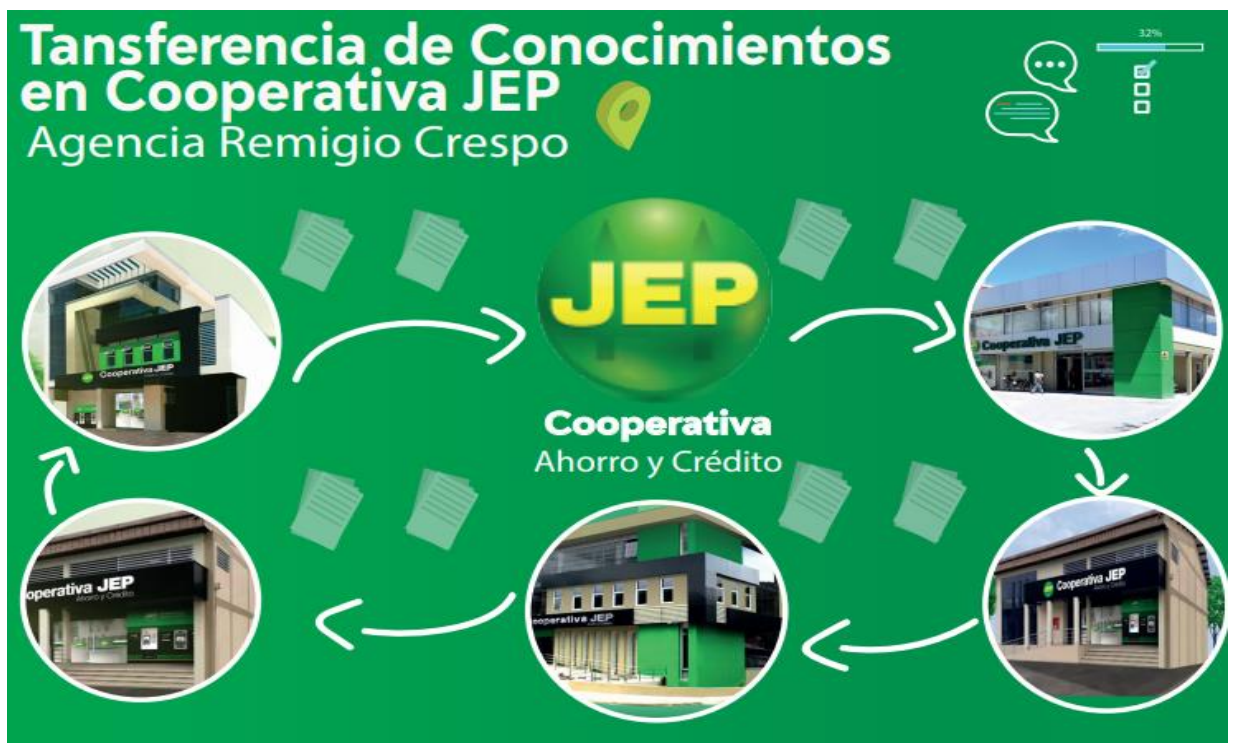

\section{Promoción:}

La publicidad se realizará a través de redes sociales, por lo tanto, se enviará imágenes de las promociones de las tarjetas de crédito y demás descuentos todo el día con varios impactos, a continuación, se presentan las diferentes opciones dentro del marketing digital. 
Revista Arbitrada Interdisciplinaria KOINONIA

Año IV. Vol IV. N8. Julio - Diciembre 2019

Hecho el depósito de Ley: FA2016000010

ISSN: $2542-3088$

FUNDACIÓN KOINONIA (F.K). Santa Ana de Coro. Venezuela.

Página web.

Figura 7.

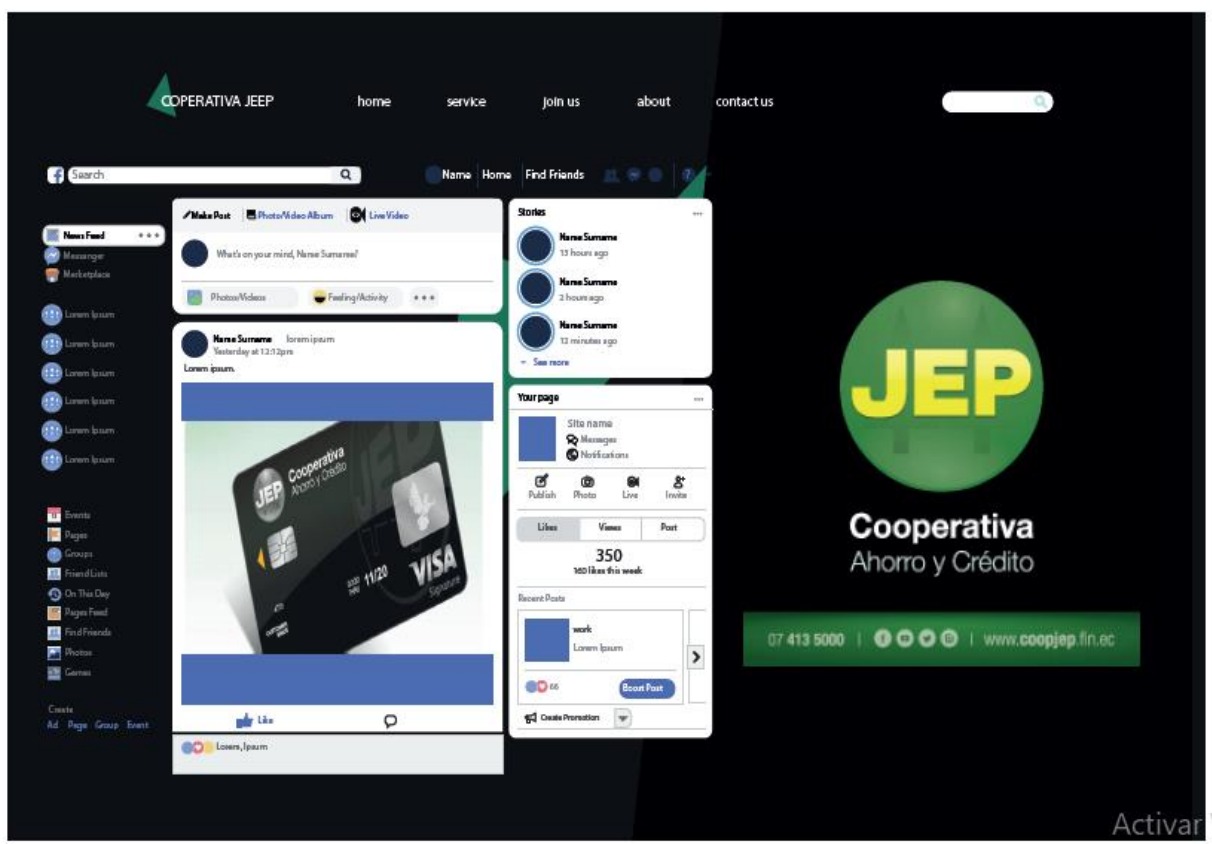

Promoción en Facebook.

Figura 8. 
Revista Arbitrada Interdisciplinaria KOINONIA

Año IV. Vol IV. N8. Julio - Diciembre 2019

Hecho el depósito de Ley: FA2016000010 ISSN: 2542-3088

FUNDACIÓN KOINONIA (F.K). Santa Ana de Coro. Venezuela.

Elizabeth Magdalena Trelles Méndez; Juan Carlos Erazo Álvarez; Cecilia Ivonne Narváez Zurito
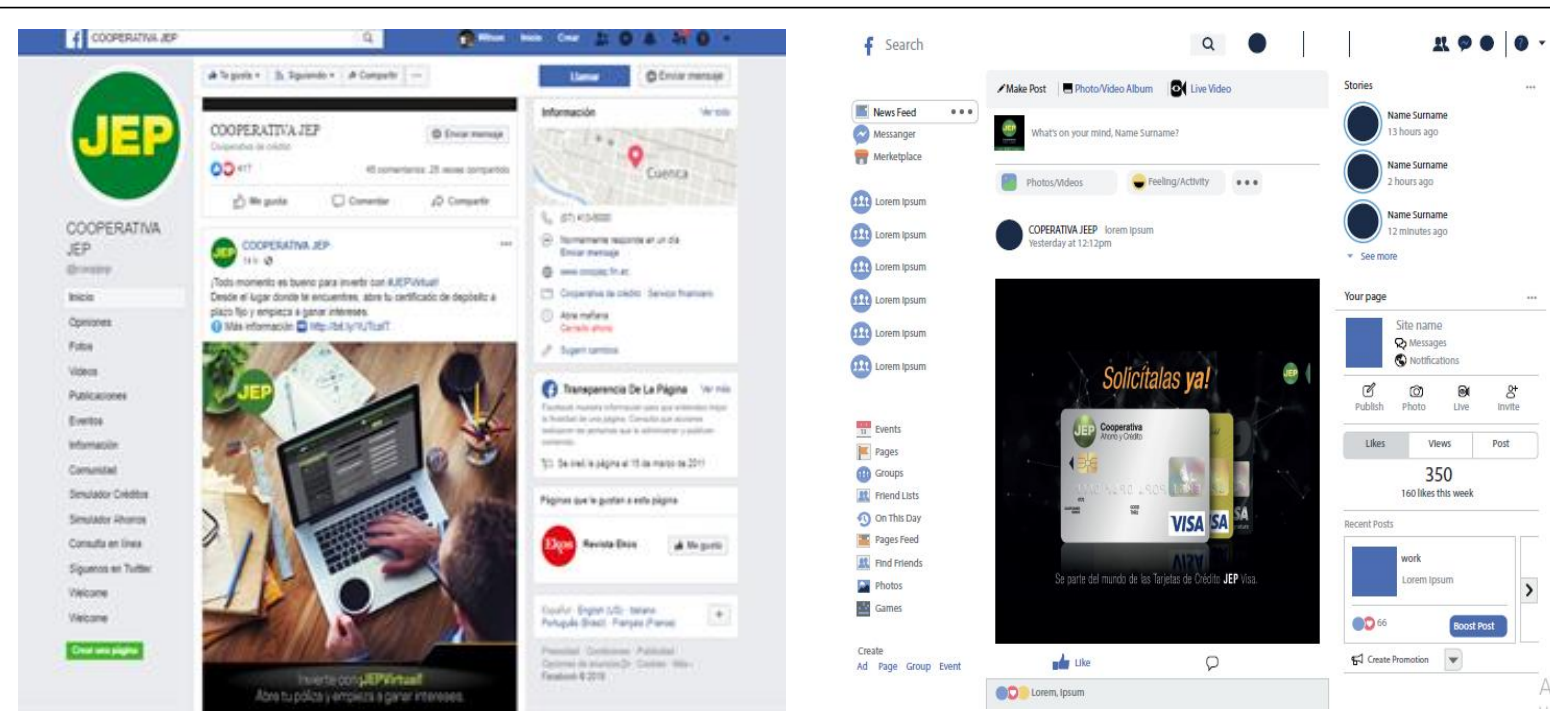

\section{Promoción en Instagram.}

Figura 9.

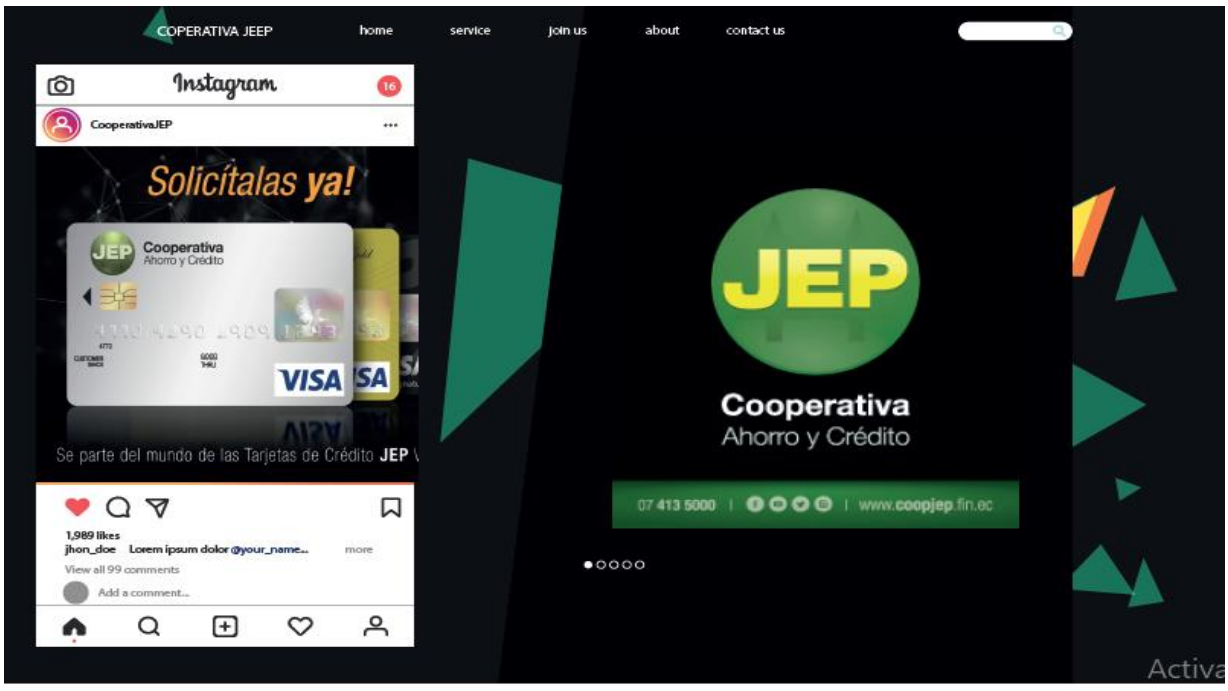

Promoción en Twitter.

Figura 10. 
Revista Arbitrada Interdisciplinaria KOINONIA

Año IV. Vol IV. N ${ }^{\circ}$ Julio-Diciembre 2019

Hecho el depósito de Ley: FA2016000010 ISSN: 2542-3088

FUNDACIÓN KOINONIA (F.K). Santa Ana de Coro. Venezuela.

Elizabeth Magdalena Trelles Méndez; Juan Carlos Erazo Álvarez; Cecilia Ivonne Narváez Zurito

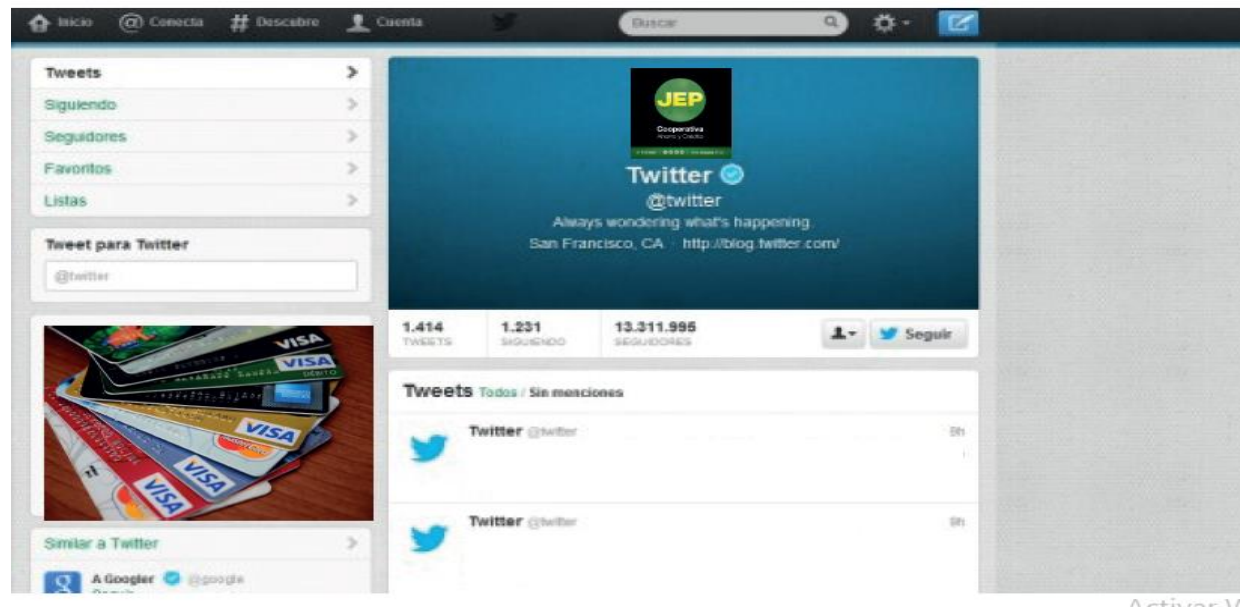

Promoción en YouTube

Figura 10. 


$\equiv$ VouTube $^{\mathrm{EC}} \quad$ Buscar

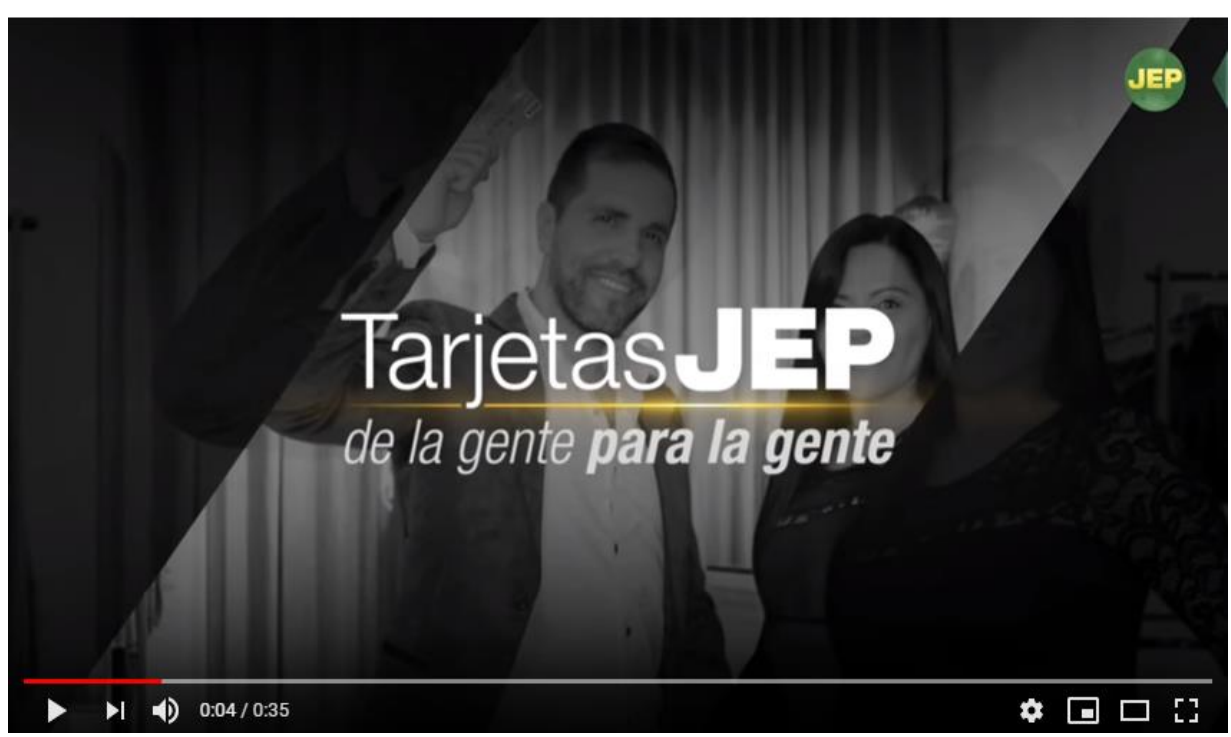

Para finalizar la promoción se sugiere realizar alianzas estratégicas con las empresas de la Ciudad de Cuenca que generan turismo y marcas importantes de referencia en la Ciudad. 
Promociones de alianzas estratégicas.

Figura 11.

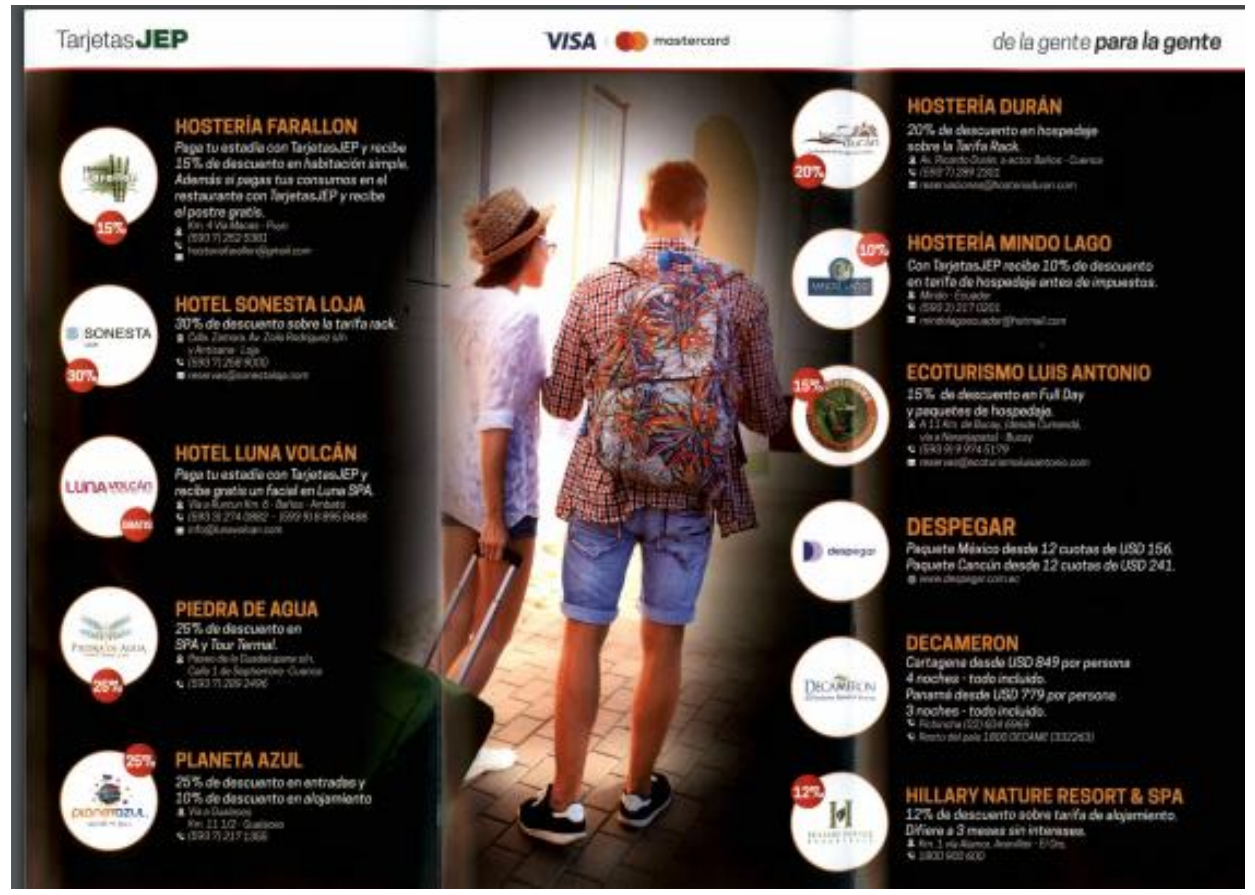

Se plantea estas alianzas estratégicas con las empresas Hostería Farallón, hostería Sonesta Loja, Hotel Luna Volcán, Piedra de Agua, Planeta Azul, Hostería Durán, Hostería Mindo Lago, Ecoturismo Luis Antonio, Despegar, Decamerón, Hillary Nature Resort, Spa, El rey del burrito, Parrillada la herradura, Mi escondite, Tacanijo entre otros, se les concederá descuentos con los pagos de las tarjetas de crédito JEP, sin embargo a cambio se les dará el pago de nómina gratis a todos los empleados de cada una de las empresas en alianza, también transacciones JEP empresas sin costo, con ello se logra posicionar mejor la marca.

\section{Precio}

La adquisición de las tarjetas de crédito tiene un costo de impresión del plástico de 4.89 dólares, el resto de costos son cero es decir mantenimiento, renovación. Los cupos disponibles son de acuerdo a la escala de ingresos de los socios y el sistema del Score de la JEP, tiene las siguientes calificaciones: 


\begin{tabular}{|l|l|l|}
\hline A-AA-AAA & rangos de & $\begin{array}{l}\$ 500,00 \text { a } \\
\$ 20000,00\end{array}$ \\
\hline Analista & rangos de & $\begin{array}{l}\$ 500,00 \text { a } \\
\$ 3000,00\end{array}$ \\
\hline Sin información & rangos de & $\begin{array}{l}\$ 500,00 \text { a } \\
\$ 2000,00\end{array}$ \\
\hline
\end{tabular}

La tasa de interés por avance es de $\$ 5$, por cada $\$ 100,00$ de avance en efectivo. Las tasas de interés que se manejan es el 17,23 \% de 3, 6, 9, 12, 24 meses efectiva. Las tasas por mora están en los rangos de cero días de mora con cero \% de cobro en mora, de 1 a 15 días es el 5\%, de 16 a 30 días es el 7\%, de 31 días a 60 días es del $9 \%$, mayores a 60 días es del 10\%.

El costo por pagos con cheques es de hasta $\$ 2,79$ máximo.

En cuanto al precio de la campaña publicitaria, se tiene el costo de la innovación a cargo del departamento de investigación, desarrollo e innovación con base en la investigación del comportamiento del consumidor y la sustentación teórica. Al cliente no se le recargará valor alguno por la innovación y la Cooperativa absorberá los beneficios de la innovación.

En consecuencia, el Mix del Marketing aplicado a la investigación es Producto, Plaza y Promoción. Por cuanto el precio queda descrito en la medida de su costo hundido y los derrames o beneficios por la innovación.

\section{CONCLUSIONES}

Se desarrolla la propuesta de un sistema de estrategias de marketing para implementar en las Agencias a nivel nacional de la Cooperativa JEP utilizando la transferencia de conocimiento, con ello se plantea este sistema de estrategias apegado al marketing mix, basado principalmente en la publicidad digital, para personas jóvenes, también se encontró en la investigación de campo que la mayoría de socios destinan el dinero para uso de capital de trabajo y en segundo lugar gastos de 
consumo, se encontró falencias del servicio en la atención y respuesta efectiva, predisposición a la atención al socio.

Se pudo evidenciar las falencias del servicio y se plantea implementar el modelo de gestión del sistema de estrategias de marketing, para dar una respuesta eficaz, generar el camino mediante el modelo hacia buenas prácticas en la administración y la gestión de los recursos disponibles para tener una óptima utilización del mismo.

\section{REFERENCIAS CONSULTADAS}

1. Álvarez Álvarez, V. A., Erazo Álvarez, J. C., Narváez Zurita, C. I., \& Erazo Álvarez, C. A. (2019). Plan de social media como estrategia de posicionamiento de marca aplicado a la empresa Importadora Zumbauto. Visionario Digital, 273.

2. Ardura, I. R. (2007). Estrategías y tecnicas de comunicación. Barcelona: Editorial UOC.

3. Chinguel y Caqui. (2017). Estrategias de marketing educacional para incrementar la demanda.

4. Córdova López, F. (2009). Del Marketing transaccional al Marketing relacional. Cali: Unilibre.

5. Espinoza, M. y. (2013). El marketing de contenidos.

6. García, V. B. (2013). Fundamentos del Marketing. Barcelona: Anglofort S.A.

7. Garcillán y Rivera, 2. (2012). Dirección de Marketing, fundamentos y aplicaciones. Madrid: ESIC Businessmarketingschool.

8. Keller, K. y. (2009). El marketing digital. México.

9. Kotler y Armstrong, 2. (2012). El marketing. México: Perarson Educación.

10. León González, V. J., Erazo Álvarez, J. C., Narváez Zurita, C. I., \& Solís Muñoz, J. B. (2019). Marketing mix de servicios de valor agregado de última milla. Valor y trascendencia de las 4 P. Visionario Digita, 154.

11. Rivera, G. y. (2012). Dirección de Marketing, fundamentos y aplicaciones. Madrid: ESIC Businessmarketingschool. 
12. Rivera, R. (2015). La evolución de las estrategias de marketing y su entorno digital. Madrid: Universidad Carlos III.

13. Sellers, a. y. (2006). Dirección de Marketing. Cottolengo. San Vicente: Club Universitario.

14. Suárez y Fernández. (2014). El marketing Comercial. México.

15. Tamayo. (2009). El proceso de la investigación científica. México: Lumisa.

16. Velásquez, M. O. (2014). Marketing conceptos y aplicaciones. Barraquilla: VERBUM.

\section{REFERENCES CONSULTED}

1. Álvarez Álvarez, V. A., Erazo Álvarez, J. C., Narváez Zurita, C. I., \& Erazo Álvarez, C. A. (2019). Social media plan as a brand positioning strategy applied to the Zumbauto Importing company. Digital Visionary, 273.

2. Ardura, I. R. (2007). Communication strategies and techniques. Barcelona: UOC Publishing.

3. Chinguel and Persimmon. (2017). Educational marketing strategies to increase demand.

4. Córdova López, F. (2009). From transactional marketing to relational marketing. Cali: Unilibre.

5. Espinoza, M. and. (2013). Content marketing

6. García, V. B. (2013). Fundamentals of Marketing. Barcelona: Anglofort S.A.

7. Garcillán and Rivera, 2. (2012). Marketing Management, fundamentals and applications. Madrid: ESIC Businessmarketingschool.

8. Keller, K. and. (2009). Digital marketing Mexico. 
9. Kotler and Armstrong, 2. (2012). Marketing Mexico: Perarson Education.

10.León González, V. J., Erazo Álvarez, J. C., Narváez Zurita, C. I., \& Solís Muñoz, J. B. (2019). Marketing mix of last mile value-added services. Value and significance of the $4 \mathrm{P}$. Visionario Digita, 154.

11.Rivera, G. and. (2012). Marketing Management, fundamentals and applications. Madrid: ESIC Businessmarketingschool.

12.Rivera, R. (2015). The evolution of marketing strategies and their digital environment. Madrid: Carlos III University.

13.Sellers, a. Y. (2006). Marketing direction. Cottolengo San Vicente: University Club.

14.Suárez and Fernández. (2014). Commercial marketing Mexico.

15.Tamayo. (2009). The process of scientific research. Mexico: Lumisa.

16.Velásquez, M. O. (2014). Marketing concepts and applications. Barraquilla: VERBUM. 